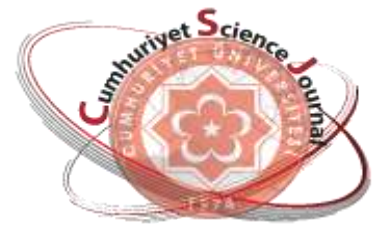

e-ISSN: $2587-246 X$

ISSN: $2587-2680$

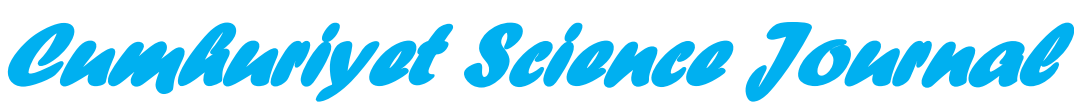

CST

Cumhuriyet Sci. J., Vol.39-3(2018) 565-572

\section{A Strongly Ill-Posed Problem for the Equation}

$$
L u \equiv x \Delta u+k\left(x,{ }^{\prime} y\right) u_{x}=x f\left(x,{ }^{\prime} y\right)
$$

\section{Mustafa YILDIZ}

Zonguldak Bülent Ecevit University, Faculty of Art and Science, Department of Mathematics, Zonguldak, TURKEY

Received: 01.08.2018; Accepted: 11.09.2018

http://dx.doi.org/10.17776/csj.450207

\footnotetext{
Abstract. In this work, we consider an inverse problem for an elliptic equation which is strongly ill-posed in Hadamard sense. We prove the uniqueness of the solution of the problem by using Carleman estimates.

Keywords: Elliptic equation, Inverse problem, Carleman estimate

$$
L u \equiv x \Delta u+k\left(x,{ }^{\prime} y\right) u_{x}=x f\left(x,{ }^{\prime} y\right)
$$

\section{Denklemi için Bir Kuvvetli Kötü Konulmuş Problem}

Özet. Bu çalışmada eliptik denklem için Hadamard anlamında kuvvetli kötü konulmuş olan bir ters problem ele alınmıştır. Bu problemin çözümünün tekliği Carleman değerlendirmeleri yardımıyla ispatlanmıştır.

Anahtar Kelimeler: Eliptik denklem, Ters problem, Carleman değerlendirmesi
}

\section{INTRODUCTION}

In this study, a strongly ill-posed inverse problem in Hadamard sense is investigated. In the domain

$$
D=\left\{(x, y) \mid x \in(0,1), y_{i} \in(0,1), i=1,2, \ldots, n\right\},
$$

we consider the equation

$$
L u \equiv x \Delta u+k u_{x}=x f\left(x,{ }^{\prime} y\right)
$$

and the conditions

$$
\left.u\right|_{y_{n}=0}=u_{0}\left(x,{ }^{\prime} y\right) ;\left.u_{y_{n}}\right|_{y_{n}=0}=u_{1}\left(x,{ }^{\prime} y\right),
$$

where ' $y=\left(y_{2}, y_{3}, \ldots, y_{n}\right)$.

Equation 1 is an elliptic equation which has important applications in potential theory, [6]. Moreover, recently, inverse and ill-posed problems for differential equations are attracting the interest of many scientists because of various applications in science and engineering such as tomography and seismology, [1-5]. 
Additional condition for the solution of Cauchy problem (1)-(2) is

$$
\left.u\right|_{y_{1}=0}=u_{2}\left(x,{ }^{\prime} y\right) .
$$

Problem 1: We now aim to get the pair $\left(u(x, y), x f\left(x,{ }^{\prime} y\right)\right)$ from equation (1) which satisfies conditions (2)-(3).

Theorem 1. Problem 1 can have only one solution in the space $C^{3}(\bar{D}) \times C^{1}(\bar{D})$ with $u \in C^{3}(\bar{D})$, $f \epsilon C^{1}(\bar{D})$.

Theorem 1 is related with the uniqueness of the solution of problem 1. In general, one can not prove the existence of solution for this problem. Because, problem (1)-(3) is strongly ill-posed in Hadamard sense. In order to prove the theorem, the following lemmas are needed.

\section{PROOF OF THE MAIN RESULT}

Let us suppose that $\left(u^{(1)}, f^{(1)}\right),\left(u^{(2)}, f^{(2)}\right)$ are two solutions for problem (1)-(3) in $C^{3}(\bar{D}) \times C^{1}(\bar{D})$. Then we have

$$
\begin{aligned}
& x \Delta u^{(i)}+k u_{x}^{(i)}=x f^{(i)}\left(x,{ }^{\prime} y\right), \\
& u^{(i)},\left.u_{y_{n}}^{(i)}\right|_{y_{n}=0}=u_{0}, u_{1}, \\
& \left.u^{(i)}\right|_{y_{1}=0}=u_{2}, i=1,2 .
\end{aligned}
$$

For $\bar{u}=u^{(2)}-u^{(1)}, \bar{f}=f^{(2)}-f^{(1)}$ we can write

$$
\begin{aligned}
& x \Delta \bar{u}+k \bar{u}_{x}=x \bar{f}\left(x,{ }^{\prime} y\right), \\
& \bar{u},\left.\bar{u}_{y_{n}}\right|_{y_{n}=0}=0, \\
& \left.\bar{u}\right|_{y_{1}=0}=0 .
\end{aligned}
$$

Taking derivative of both sides of equation (7) with respect to $y_{1}$, we get

$$
x \Delta \bar{u}_{y_{1}}+k \bar{u}_{y_{1} x}=0 .
$$

In equation (10), if we take $w=\bar{u}_{y_{1}}$ we have

$$
x \Delta w+k w_{x}=0
$$

and conditions (8) become

$$
w,\left.w_{y_{n}}\right|_{y_{n}=0}=0 .
$$

Let us first consider the following general problem,

$$
\begin{aligned}
& \left|\Delta_{x, y} z\right| \leq C(|z|+|\nabla z|), \\
& z\left(x,,^{\prime} y, 0\right)=0, z_{y_{n}}(x, y, 0)=0 .
\end{aligned}
$$


It is easy to prove that there is only zero solution of problem (11)-(12) in $C^{2}(\bar{D})$ if (13)-(14) has only zero solution in $C^{2}(\bar{D})$.

We now attempt to prove that problem (13)-(14) can have zero solution in $C^{2}(\bar{D})$ using the Carleman method.

For the sake of simplicity we have

$$
y_{n}=u_{1}, x=u_{2}, y_{i}=u_{2+i}, i=1,2,3, \ldots, n-1 .
$$

Let

$$
\Omega\left(u^{0}\right)=\left\{u: u_{1}>0,0<\delta u_{1}<\gamma-\frac{1}{2} \sum_{i=2}^{n+1}\left(u_{i}-u_{i}^{0}\right)^{2}, \delta>1, \gamma<1\right\}
$$

and

$$
\psi(u)=\delta u_{1}+\frac{1}{2} \sum_{i=2}^{n+1}\left(u_{i}-u_{i}^{0}\right)^{2}+\alpha_{0}, \alpha_{0}>0, \gamma+\alpha_{0}=\eta<1 .
$$

If we choose

$$
u^{0}=\left(u_{2}^{0}, u_{3}^{0}, \ldots, u_{n+1}^{0}\right) \epsilon D^{1}, D^{1}=\left\{(x, y):\left(x, y_{1}, \ldots, y_{n-1}\right) \epsilon(0,1)^{n}\right\},
$$

then it is clear that

$$
\alpha_{0}<\psi(u)<\eta \text { in the domain }\left(0, u^{0} \epsilon \Omega\left(u^{0}\right)\right) \subset D \text { and } \Omega\left(u^{0}\right) .
$$

Let $H=\exp \left(\lambda \psi^{-v}\right)$, where $\lambda, v$ are positive parameters.

Lemma 1. For every $\varphi \epsilon C^{2}(\bar{D})$

$$
\begin{gathered}
-\varphi \Delta \varphi H^{2}=\left[|\nabla \varphi|^{2}-\sum_{i=1}^{n+1}\left(2 \lambda^{2} v^{2} \psi^{-2 v-2} \psi_{u_{i}}^{2}+\lambda \nu(v+1) \psi^{-v-2} \psi_{u_{i}}^{2}+\lambda v \psi^{-v-1} \psi_{u_{i} u_{i}}\right) \varphi^{2}\right] H^{2} \\
+d_{1}(\varphi)
\end{gathered}
$$

where

$$
d_{1}(\varphi)=-\sum_{i=1}^{n+1}\left(\varphi \varphi_{u_{i}} H^{2}+\psi^{-v-1} \lambda \nu \psi_{u_{i}} \varphi^{2} H^{2}\right)_{u_{i}}
$$

Proof. From the definition, we have

$$
-\varphi \Delta \varphi H^{2}=-\varphi\left(\sum_{i=1}^{n+1} \varphi_{u_{i} u_{i}}\right) e^{2 \lambda \psi^{-v}} .
$$

On the other hand,

$$
-\varphi \varphi_{u_{i} u_{i}} H^{2}=-\left(\varphi \varphi_{u_{i}} e^{2 \lambda \psi^{-v}}\right)_{u_{i}}+\varphi_{u_{i}}^{2} e^{2 \lambda \psi^{-v}}-2 \lambda v \varphi \varphi_{u_{i}} e^{2 \lambda \psi^{-v}} \psi_{u_{i}} \psi^{-v-1}
$$

and

$$
\begin{aligned}
& -2 \lambda v \psi^{-v-1} \psi_{u_{i}} H^{2} \varphi \varphi_{u_{i}}=-\left(\lambda v \psi_{u_{i}} \psi^{-v-1} H^{2} \varphi^{2}\right)_{u_{i}}-2 \lambda^{2} v^{2} \psi_{u_{i}}^{2} \psi^{-2 v-2} H^{2} \varphi^{2} \\
& -\lambda v(v+1) \psi_{u_{i}}^{2} H^{2} \psi^{-v-2} \varphi^{2}+\lambda v \psi^{-v-1} \psi_{u_{i} u_{i}} H^{2} \varphi^{2}, i=1,2,3, \ldots, n+1 .
\end{aligned}
$$


By the last two equations, (15) is obtained.

\section{Lemma 2.}

For

$$
v_{0}=\frac{(n+2) \eta}{\delta(1-\eta)}, \lambda_{0}=2(v+2)^{2}\left(\delta^{2}+\gamma^{2}\right)^{2}+2,
$$

if $v \geq 2+v_{0}, \lambda \geq \lambda_{0}$, then for any $\varphi \epsilon C^{2}(\bar{D})$ the inequality

$$
\psi^{v+1}(\Delta \varphi)^{2} H^{2} \geq 3 \lambda^{3} v^{4} \delta^{4} \psi^{-2 v-2} H^{2} \varphi^{2}-2 \lambda v|\nabla \varphi|^{2} H^{2}+d_{2}(\varphi)
$$

is valid, where

$$
\begin{aligned}
d_{2}(\varphi H)= & \sum_{i=1}^{4} d_{2 i}(\varphi H) \\
d_{21}(\varphi H)= & 4 \lambda v \sum_{i, j=1}^{n+1}\left[\psi_{u_{i}}\left(\varphi_{u_{i}}-\lambda v \psi_{u_{i}} \psi^{-v-1} \varphi\right)\left(\varphi_{u_{i}}-\lambda v \psi_{u_{i}} \psi^{-v-1} \psi\right)\right]_{u_{j}} \\
& -2 \lambda v \sum_{i, j=1}^{n+1}\left[\psi_{u_{i}}\left(\varphi_{u_{i}}-\lambda v \psi_{u_{i}} \psi^{-v-1} \varphi\right)\right]_{u_{i}}+2 v^{2} \lambda^{2} \sum_{i=1}^{n+1}\left(\psi^{-v-1} \psi_{u_{i}} \varphi^{2}\right)_{u_{i}}{ }^{\prime} \\
d_{22}(\varphi H)= & 2 \lambda^{3} v^{3}\left(\psi^{-2 v-2} \varphi^{2}|\nabla \psi|^{2} \sum_{i=1}^{n+1} \psi_{u_{i}}\right)_{u_{i}}, \\
d_{23}(\varphi H)= & -2 \lambda^{2} v^{2}(v+1) \sum_{i=1}^{n+1}\left(|\nabla \psi|^{2} \psi^{-v-2} \psi_{u_{i}} \varphi^{2}\right)_{u_{i}}, \\
d_{24}(\varphi H)= & 2 \lambda^{2} v^{2} \sum_{i=1}^{n+1}\left(\psi_{u_{i}} \psi^{-v-1} \varphi^{2}\right)_{u_{i}} .
\end{aligned}
$$

Proof. For the auxiliary function $w=H \varphi$, we have

$$
\begin{aligned}
\varphi_{u_{i} u_{i}}= & H^{-1}\left(w_{u_{i} u_{i}}+2 \lambda v \psi_{u_{i}} \psi^{-v-1} w_{u_{i}}+\lambda v \psi^{-v-1} \psi_{u_{i} u_{i}} w-\lambda v(v+1) \psi_{u_{i}}^{2} \psi^{-v-2} w\right. \\
& \left.+\lambda^{2} v^{2} \psi^{-2 v-2} w \psi_{u_{i}}^{2}\right)
\end{aligned}
$$

Multiplying both sides of the last expression with $\mathrm{H}$ and adding from 1 to $(\mathrm{n}+1)$ with respect to $\mathrm{i}$, we obtain

$$
\begin{aligned}
H^{2}(\Delta \varphi)^{2}= & \left(\sum_{i=1}^{n+1} w_{u_{i} u_{i}}+2 \lambda v \psi^{-v-1} \sum_{i=1}^{n+1} \psi_{u_{i}} w_{u_{i}}+\lambda v \psi^{-v-1} w \sum_{i=1}^{n+1} \psi_{u_{i} u_{i}}\right. \\
& \left.-\lambda v(v+1) w \psi^{-v-2} \sum_{i=1}^{n+1} \psi_{u_{i}}^{2}+\lambda^{2} v^{2} \psi^{-2 v-2} w \sum_{i=1}^{n+1} \psi_{u_{i}}^{2}\right)^{2}
\end{aligned}
$$

The both hand sides of the last equality are multiplied with $\psi^{v+1}$, we get

$$
\begin{aligned}
\psi^{v+1} H^{2}(\Delta \varphi)^{2}= & \psi^{v+1}\left(\Delta w+\sum_{i=1}^{n+1}\left(\lambda^{2} v^{2} \psi^{-2 v-2} \psi_{u_{i}}^{2}\right.\right. \\
& \left.\left.-\lambda v(v+1) \psi^{-v-2} \psi_{u_{i}}^{2}+\lambda v \psi^{-v-1} \psi_{u_{i} u_{i}}\right) w+2 \lambda v \psi^{-v-1} \sum_{i=1}^{n+1} \psi_{u_{i}} w_{u_{i}}\right)^{2} .
\end{aligned}
$$

Having

$$
a=\Delta w+\sum_{i=1}^{n+1}\left(\lambda^{2} v^{2} \psi^{-2 v-2} \psi_{u_{i}}^{2}-\lambda v(v+1) \psi^{-v-2} \psi_{u_{i}}^{2}+\lambda v \psi^{-v-1} \psi_{u_{i} u_{i}}\right) w,
$$




$$
b=2 \lambda v \psi^{-v-1} \sum_{i=1}^{n+1} \psi_{u_{i}} w_{u_{i}}
$$

and applying the inequality $(a+b)^{2} \geq 2 a b$ to the last expression, it is obvious that

$$
\begin{gathered}
\psi^{v+1} H^{2}(\Delta \varphi)^{2}=\psi^{v+1}\left(\Delta w+\sum_{i=1}^{n+1}\left(\lambda^{2} v^{2} \psi^{-2 v-2} \psi_{u_{i}}^{2}\right.\right. \\
\left.\left.-\lambda v(v+1) \psi^{-v-2} \psi_{u_{i}}^{2}+\lambda v \psi^{-v-1} \psi_{u_{i} u_{i}}\right) w+2 \lambda v \psi^{-v-1} \sum_{i=1}^{n+1} \psi_{u_{i}} w_{u_{i}}\right)^{2} \\
\geq 4 \lambda v\left(\sum_{i=1}^{n+1} \psi_{u_{i}} w_{u_{i}}\right)\left[\Delta w+\sum_{j=1}^{n+1} \lambda v \psi^{-v-1}\left(\lambda v \psi^{-v-1} \psi_{u_{j}}^{2}\right.\right. \\
\left.\left.-(v+1) \psi^{-1} \psi_{u_{j}}^{2}+\psi_{u_{j} u_{j}}\right) w\right] .
\end{gathered}
$$

Now let us consider each term in (17) respectively:

1. $4 \lambda v \sum_{i, j=1}^{n+1} \psi_{u_{i}} w_{u_{i}} w_{u_{j} u_{j}}=4 \lambda v \sum_{i, j=1}^{n+1}\left(\psi_{u_{i}} w_{u_{j}} w_{u_{i}}\right)_{u_{j}}-4 \lambda v \sum_{i, j=1}^{n+1} \psi_{u_{i}} w_{u_{j}} w_{u_{i} u_{j}}$

$$
=4 \lambda v \sum_{i, j=1}^{n+1}\left(\psi_{u_{i}} w_{u_{j}} w_{u_{i}}\right)_{u_{j}}-2 \lambda v \sum_{i, j=1}^{n+1}\left(\psi_{u_{i}} w_{u_{j}}^{2}\right)_{u_{i}}+2 \lambda v \sum_{i=1}^{n+1} w_{u_{i}}^{2}
$$

Additionally, since

$$
\begin{aligned}
& w=\varphi H=e^{\lambda \psi^{-v}} \varphi, \\
& w_{u_{i}}=H\left(\varphi_{u_{i}}-\lambda v \psi^{-v-1} \psi_{u_{i}} \varphi\right), v \geq 2, \delta>1
\end{aligned}
$$

then,

$$
\begin{aligned}
4 \lambda v \sum_{i, j=1}^{n+1} \psi_{u_{i}} w_{u_{i}} w_{u_{j} u_{j}}=4 \lambda v \sum_{i, j=1}^{n+1}\left[\psi_{u_{i}} H\left(\varphi_{u_{i}}-\lambda v \psi^{-v-1} \psi_{u_{i}} \varphi\right) H\left(\varphi_{u_{j}}-\lambda v \psi^{-v-1} \psi_{u_{j}} \varphi\right)\right]_{u_{j}} \\
-2 \lambda v \sum_{i, j=1}^{n+1}\left[\psi_{u_{i}} H^{2}\left(\varphi_{u_{j}}-\lambda v \psi^{-v-1} \psi_{u_{j}} \varphi\right)^{2}\right]_{u_{j}}+2 \lambda v \sum_{i=1}^{n+1} H^{2}\left(\varphi_{u_{j}}-\lambda v \psi^{-v-1} \psi_{u_{j}} \varphi\right)^{2} \\
=d_{21}(H \varphi)+2 \lambda v|\nabla \varphi|^{2} H^{2}|\nabla \psi|^{2}+2 \lambda^{3} v^{3} \psi^{-2 v-2} H^{2} \varphi^{2}|\nabla \psi|^{2} \\
-2 \lambda^{2} v^{2} \sum_{i=1}^{n+1}\left(H^{2} \psi^{-v-1} \varphi^{2} \psi_{u_{i}}\right)_{u_{i}}-2 \lambda^{2} v^{2}(v+1) \varphi^{2} \psi^{-v-2} H^{2}|\nabla \psi|^{2}+2 \lambda^{2} v^{2} \varphi^{2} \psi^{-v-1} H^{2} \\
\geq d_{21}(H \varphi)+2 \lambda v|\nabla \varphi|^{2} H^{2}+2 \lambda^{3} v^{3} \psi^{-2 v-2}|\nabla \psi|^{2} \varphi^{2} H^{2} .
\end{aligned}
$$

2. From the conditions $|\nabla \psi| \geq \delta>1, \eta^{-1}<\psi^{-1}, v>\frac{(n+2) \eta}{\delta(1-\eta)}$, we have the inequality is

$$
\begin{aligned}
& \psi^{-1}-\frac{n+2}{2 v}|\nabla \psi|^{-2}>1 \text { and } \\
& 4 \lambda v \sum_{i, j=1}^{n+1} \psi_{u_{i}} w_{u_{i}} \lambda^{2} v^{2} \psi^{-2 v-2} \psi_{u_{j}}^{2} w=2 \lambda^{3} v^{3} \sum_{i, j=1}^{n+1}\left(\psi_{u_{i}} \psi_{u_{j}}^{2} \psi^{-2 v-2} w^{2}\right)_{u_{i}} \\
& \quad+4 \lambda^{3} v^{3}(v+1) \psi^{-2 v-3}|\nabla \psi|^{4} w^{2}-2 \lambda^{3} v^{3} \psi^{-2 v-2}|\nabla \psi|^{2} w^{2}-4 \lambda^{3} v^{3} \psi^{-2 v-2}\left(\sum_{i=2}^{n+1} \psi_{u_{i}}^{2}\right) w^{2} \\
& \quad \geq d_{22}(w)+4 \lambda^{3} v^{4} \psi^{-2 v-2}|\nabla \psi|^{4} w^{2}\left(\psi^{-1}-\frac{n+1}{2 v}|\nabla \psi|^{-2}\right)
\end{aligned}
$$




$$
\begin{aligned}
& \geq d_{22}(w)+4 \lambda^{3} v^{4} \delta^{4} \psi^{-2 v-2} w^{2} \\
& =d_{22}(H \varphi)+4 \lambda^{3} v^{4} \delta^{4} \psi^{-2 v-2} \varphi^{2} H^{2} .
\end{aligned}
$$

3.

$$
\begin{aligned}
-4 \lambda v \sum_{i, j=1}^{n+1} \psi_{u_{i}} w_{u_{i}} \lambda v(v+1) \psi^{-v-2} \psi_{u_{j}}^{2} w=-2 \lambda^{2} v^{2}(v+1) \sum_{i, j=1}^{n+1}\left(\psi_{u_{i}} \psi_{u_{j}}^{2} \psi^{-v-2} w^{2}\right)_{u_{i}} \\
\quad+2 \lambda^{2} v^{2}(v+1) \psi^{-v-2}|\nabla \psi|^{2} w^{2}+4 \lambda^{2} v^{2}(v+1)\left(\sum_{i=2}^{n+1} \psi_{u_{i}}^{2}\right) \psi^{-v-2} w^{2} \\
-2 \lambda^{2} v^{2}(v+2)(v+1) \psi^{-v-3}|\nabla \psi|^{4} w^{2} \\
\geq d_{23}(w)-2 \lambda^{2} v^{2}(v+2)(v+1) \psi^{-v-3}|\nabla \psi|^{4} w^{2} \\
=d_{23}(H \varphi)-2 \lambda^{2} v^{2}(v+1)(v+2) \psi^{-v-3}|\nabla \psi|^{4} \varphi^{2} H^{2} .
\end{aligned}
$$

4.

$$
\begin{aligned}
4 \lambda v \sum_{i, j=1}^{n+1} \psi_{u_{i}} w_{u_{i}} \lambda v \psi^{-v-1} \psi_{u_{i} u_{j}} w=2 \lambda^{2} v^{2} \sum_{i=1}^{n+1}\left(\psi_{u_{i}} \psi^{-v-1} w^{2}\right)_{u_{i}}-2 \lambda^{2} v^{2} \psi^{-v-1} w^{2} \\
\quad+2 \lambda^{2} v^{2}(v+1) \psi^{-v-2}|\nabla \psi|^{2} w^{2} \\
\geq d_{24}(w)-2 \lambda^{2} v^{2} \psi^{-v-1} w^{2} \\
=d_{24}(H \varphi)-2 \lambda^{2} v^{2} \psi^{-v-1} \varphi^{2} H^{2}
\end{aligned}
$$

By inequalities (17)-(21), we obtain

$$
\begin{aligned}
\psi^{v+1} H^{2}(\Delta \varphi)^{2} & \geq d_{2}(\varphi H)+2 \lambda v|\nabla \varphi|^{2} H^{2} \\
& +\lambda^{2} v^{2} \varphi^{2} H^{2}\left(4 \lambda v^{2} \delta^{4} \psi^{-v-1}-2-2(v+1)(v+2) \psi^{-2}|\nabla \psi|^{4}\right) \psi^{-v-1}
\end{aligned}
$$

Since

$$
\lambda v^{2} \delta^{4} \psi^{-v-1}-2(v+1)(v+2) \psi^{-2}|\nabla \psi|^{4}-2 \geq 0
$$

for $\lambda \geq \lambda_{0}$, then (16) results from inequality (22). Thus Lemma 2 is proven.

\section{Lemma 3.}

Let the conditions of Lemma 2 be satisfy and let

$$
v \geq v_{1}=\max \left\{v_{0}+n+1,4(n+1)+8(n+1)\left(\delta^{2}+\eta^{2}\right)+1\right\} .
$$

Then

$$
\begin{aligned}
&-2(n+1) \lambda v \varphi(\Delta \varphi) H^{2}+\psi^{v+1}(\Delta \varphi)^{2} H^{2} \\
& \geq 2 \lambda^{3} v^{3} \psi^{-2 v-2} \varphi^{2} H^{2}+2 \lambda v n|\nabla \varphi|^{2} H^{2}+2(n+1) \lambda v d_{1}(\varphi)+d_{2}(\varphi) .
\end{aligned}
$$

\section{Proof.}

Multiplying equation (15) with $2(n+1) \lambda v$ and adding the resulting expression with (16), one gets $-2(n+1) \lambda v \varphi(\Delta \varphi) H^{2}+\psi^{v+1}(\Delta \varphi)^{2} H^{2}$ 


$$
\begin{aligned}
& \geq 2 \lambda v n|\nabla \varphi|^{2} H^{2}+\left(3 \lambda^{3} v^{4} \delta^{4} \psi^{-2 v-2}-4(n+1) \lambda^{3} v^{3} \delta^{3} \psi^{-2 v-2}|\nabla \psi|^{2}\right. \\
& \left.-2(n+1) \lambda^{2} v^{2}(v+1) \psi^{-v-2} \sum_{i=1}^{n+1} \psi_{u_{i}}^{2}+2(n+1) \lambda^{2} v^{2} \psi^{-v-1}\right) \varphi^{2} H^{2} \\
& +2(n+1) \lambda v d_{1}(\varphi)+d_{2}(\varphi) .
\end{aligned}
$$

Since $v \geq v_{1}$, (23) is obtained from the last inequality. This proves Lemma 3 .

\section{Proof of Theorem 1.}

Considering Lemma 3 and inequality (13), we have

$$
\begin{aligned}
{\left[(n+1)^{2}\right.} & \left.\lambda^{2} v^{2} z^{2}+2 c^{2}\left(z^{2}+|\nabla z|^{2}\right)\right] H^{2}+2 \psi^{v+1} H^{2}\left(|z|^{2}+|\nabla z|^{2}\right) C^{2} \\
& \geq\left((n+1)^{2} \lambda^{2} v^{2} z^{2}+(\Delta z)^{2}\right) H^{2}+\psi^{v+1}(\Delta z)^{2} H^{2} \\
& \geq 2(n+1) \lambda v|z||\Delta z| H^{2}+(\Delta z)^{2} H^{2} \psi^{v+1} \\
& \geq-2(n+1) \lambda v|z||\Delta z| H^{2}+\psi^{v+1} H^{2}(\Delta z)^{2} \\
& \geq-2 \lambda^{3} v^{3} \psi^{-2 v-2} z^{2} H^{2}+2 \lambda v n|\nabla z|^{2} H^{2}+2(n+1) \lambda v d_{1}(\varphi)+d_{2}(\varphi)
\end{aligned}
$$

or

$$
\begin{aligned}
0 \geq H^{2} z^{2}\left(2 \lambda^{3} v^{3} \psi^{-2 v-2}-\left[(n+1)^{2} \lambda^{2} v^{2}+2 C^{2}+2 \psi^{v+1} C^{2}\right]\right. \\
+H^{2}|\nabla z|^{2}\left(2 \lambda v n-2 C^{2} \psi^{v+1}-2 C^{2}\right)+2(n+1) \lambda v d_{1}(\varphi)+d_{2}(\varphi) .
\end{aligned}
$$

If we take $v \geq v_{1}$, then it is clear that there exists $\overline{\lambda_{0}}$ such that

$$
\begin{aligned}
& 2 \lambda^{3} v^{3} \psi^{-2 v-2}-\left[(n+1)^{2} \lambda^{2} v^{2}+2 C^{2}+2 \psi^{v+1} C^{2}\right] \geq \lambda, \\
& 2 \lambda v-2 C^{2} \psi^{v+1}-2 C^{2} \geq \lambda
\end{aligned}
$$

for $\lambda>\overline{\lambda_{0}}$.

In addition, in the domain $\Omega\left(u^{0}\right), H \geq 1$. Then in $\Omega\left(u^{0}\right)$ for $\lambda \geq \overline{\lambda_{0}}$, it is possible to write the following inequality from (24):

$$
0 \geq \lambda\left(z^{2}+|\nabla z|^{2}\right)+4 \lambda v d_{1}(z H)+d_{2}(z H) .
$$

Integrating (25) over $\Omega\left(u^{0}\right)$ and taking the limit for $\lambda \rightarrow \infty$ of the resulting inequality, then from the definition of the function $\mathrm{H}$ and conditions (14) we have

$$
\int_{\Omega\left(u^{0}\right)}\left(z^{2}+|\nabla z|^{2}\right) d \Omega\left(u^{0}\right) \leq 0 .
$$

That is, in the domain $\Omega\left(u^{0}\right)$, we get $z \equiv 0$.

Varying the point $u^{0}$ at the boundary of the domain $\mathrm{D}$ where $\left\{y_{n}=0\right\}$, one can prove that $z=0$ in the set of points which satisfy the condition $0 \leq y_{n} \leq \delta$.

Similarly, we can prove that $z=0$ for the remaining part of $D$. 
To complete the proof of theorem 1, it is necessary to show that every solution of problem (11)-(12) satisfies the conditions of problem (13)-(14) at $D$ for points $x>0$.

Obviously each solution of (11) satisfies inequality (13) in the domain $\Omega\left(u^{0}\right)$ and for $C$ dependent on the constant $k$. A similar case is valid for conditions (12)-(14). That is, the solution $w$ of problem (11)-(12) satisfies conditions (13)-(14). Then, $w \equiv 0$ in the domain $D$. Moreover, $\bar{u} \equiv 0$ in $D$ from the equality $w=$ $\bar{u}_{y_{1}}$ and condition (9). On the other hand we see that $f \equiv 0$ from (7). Thus theorem 1 is proven.

\section{REFERENCES}

[1]. Amirov, A. Kh., Integral Geometry and Inverse Problems for Kinetic Equations. Utrecht, The Netherlands: VSP, 2001.

[2]. Gölgeleyen, F. and Yamamoto, M., An inverse problem for the Vlasov-Poisson system. Journal of Inverse and Ill-posed Problems, 23-4 (2015) 363-372.

[3]. Gölgeleyen, I., An integral geometry problem along geodesics and a computational approach. An. Univ.“Ovidius” Constanţa, Ser. Mat, 18-2 (2010) 91-112.

[4]. Lavrent'ev, M. M., Some Improperly Posed Problems of Mathematical Physics. New York: Springer-Verlag, 1967.

[5]. Lavrent'ev, M. M., Romanov, V. G. and Shishatskii, S. P., Ill-Posed Problems of Mathematical Physics and Analysis. Providence: American Mathematical Society, 1986.

[6]. Mikhailov, V.P., Partial Differential Equations. 2nd ed. Moscow: Mir Publishers, 1978. 